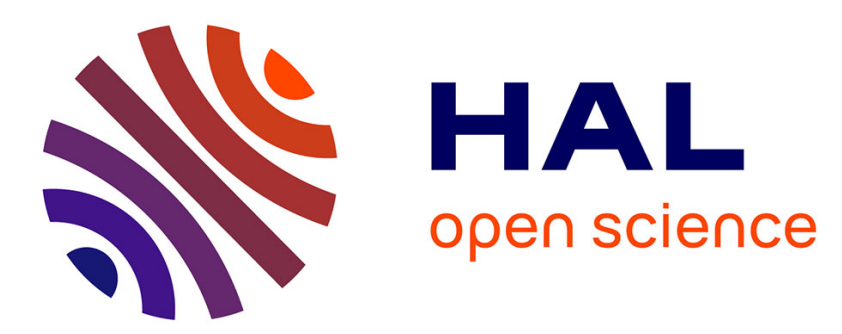

\title{
Axisymmetric wave propagation in multilayered poroelastic grounds due to a transient acoustic point source
}

Julien Capeillère, Arnaud Mesgouez, Gaëlle Lefeuve-Mesgouez

\section{To cite this version:}

Julien Capeillère, Arnaud Mesgouez, Gaëlle Lefeuve-Mesgouez. Axisymmetric wave propagation in multilayered poroelastic grounds due to a transient acoustic point source. Soil Dynamics and Earthquake Engineering, 2013, 52 (September 2013), pp.70-76. 10.1016/j.soildyn.2013.05.003 . hal00734083v5

\section{HAL Id: hal-00734083 \\ https://hal.science/hal-00734083v5}

Submitted on 2 Jul 2013

HAL is a multi-disciplinary open access archive for the deposit and dissemination of scientific research documents, whether they are published or not. The documents may come from teaching and research institutions in France or abroad, or from public or private research centers.
L'archive ouverte pluridisciplinaire HAL, est destinée au dépôt et à la diffusion de documents scientifiques de niveau recherche, publiés ou non, émanant des établissements d'enseignement et de recherche français ou étrangers, des laboratoires publics ou privés. 


\title{
Axisymmetric wave propagation in multilayered poroelastic grounds due to a transient acoustic point source
}

\author{
Julien Capeillère ${ }^{\mathrm{a},}$, Arnaud Mesgouez ${ }^{\mathrm{a}, *}$, Gaëlle Lefeuve-Mesgouez ${ }^{\mathrm{a}}$, \\ ${ }^{a}$ Université d'Avignon et des Pays de Vaucluse, UMR EMMAH, \\ Faculté des Sciences, 33 rue Louis Pasteur, F-84914 Avignon, France
}

\begin{abstract}
This paper deals with the study of axisymmetric wave propagation in various acoustic / porous stratified media coupling configurations. It presents the theoretical developments of a semi-analytical method, its validation for a limit test-case half-space ground, and an extension to a realistic multilayered seabed, when spherical waves are emitted from a transient point source in water.
\end{abstract}

Keywords: Stratified poroelastic seabed, Spherical acoustic wave, Axisymmetric geometry, Hankel-Fourier transforms.

1. Introduction

2 The study of wave propagation in seawater-seabed coupling configurations 3 is of interest for underwater acoustics and civil engineering $[1,2,3]$. On 4 the one hand, the acoustic equation models the physical phenomenon in the 5 seawater part, and on the other hand, the Biot equations describe the seabed

\footnotetext{
*Corresponding author

Email address: arnaud.mesgouez@univ-avignon.fr (Arnaud Mesgouez)
} 
part $[4,5]$. In such problems, several boundary conditions between the fluid and the top porous layer can be used to model hydraulic exchanges $[1,6,7]$. The proposed study focuses on transient wave propagation in a multi-region medium composed of a fluid half-space representing seawater over a stratified poroelastic medium representing the seabed. The source is located within the seawater part and emits spherical transient waves. The purpose is to provide a semi-analytical approach to solve this coupled problem in an axisymmetric configuration.

Configurations are often restricted to 2D Cartesian geometries. Threedimensional Green's function in axisymmetric configurations was first developed by [8] for an acoustic point source located near a half-space poroelastic seabed. Nevertheless, the study was restricted to half-space situations.

Focusing on the stratified aspect of the problem, the strategies usually adopted are based on transfer matrix, stiffness matrix or transmission and reflexion matrix methods. The main difficulty deals with the conditioning of matrices, that can be overcome using specific techniques [9]. These methods historically developed for electromagnetic and then viscoelastic problems have been extended to poroelastic media. These developments have been proposed for 2D Cartesian geometries with a free surface $[9,10]$ and then with a coupling with a seawater interaction [11]. In the present article, we propose to extend the previous work to an axisymmetric geometry and to couple the stratified poroelastic medium to a fluid one. Thus, this work can be used as benchmark solutions for numerical approaches, or for comparisons with experimental results. Moreover it represents the first step of the Boundary Element Method. The axisymmetric approach is based on Hankel-Fourier 
transforms, providing thus an analytical matrix system for the fluid pressure / stresses / displacements / velocities in the frequency-wavenumber domain. To obtain results in the time and space domain, integrations are then performed numerically.

The paper is organized as follows. Section 2 describes the geometry under study, and proposes analytical solutions to the acoustic equation and Biot equations in the context of multilayered medium and axisymmetric geometry. Section 3 presents a test case to validate the results by a comparison with those of [8], and illustrates both the stratified aspect of the ground and the interface with the seawater.

\section{Model formulation}

\subsection{Geometry under study}

The configuration under investigation is a fluid half-space $\Omega_{0}$ over a stack of homogeneous and isotropic poroelastic layers $\Omega_{n}(n=1, \cdots, N)$, as shown in Fig. 1. The $z$ geometrical axis points upward. The $N$ plane and parallel interfaces are located at $z_{n} \leq 0$, with $z_{0}=0$. An acoustic point source $\mathrm{O}_{\mathrm{s}}\left(r_{\mathrm{s}}=0 ; z_{\mathrm{s}}>0\right)$ in the fluid emits transient spherical waves.

\subsection{Multilayered porous medium}

The poroelastic media are modeled using the Biot theory $[1,4,5]$. For homogeneous and isotropic layers, the physical parameters do not depend on the spatial coordinates and can be listed as follows: for the saturating fluid, dynamic viscosity $\eta$ and density $\rho_{\mathrm{f}}$; for the elastic skeleton, density $\rho_{\mathrm{s}}$ and shear modulus $\mu$ as well as connected porosity $\phi$, tortuosity $a$, absolute 
permeability $\kappa$, Lamé coefficient of the dry matrix $\lambda$, and two Biot coefficients $\beta$ and $m$. Based on the constitutive equations and the conservation of momentum in porous media, one obtains

$$
\left\{\begin{array}{l}
\sigma=(\lambda \nabla \cdot \mathbf{u}-\beta p) \mathbf{I}+2 \mu \varepsilon, \\
p=-m(\beta \nabla \cdot \mathbf{u}+\nabla \cdot \mathbf{w}), \\
\nabla \sigma=\rho \ddot{\mathbf{u}}+\rho_{\mathrm{f}} \ddot{\mathbf{w}}, \\
-\nabla p=\rho_{\mathrm{f}} \ddot{\mathbf{u}}+\frac{a \rho_{\mathrm{f}}}{\phi} \ddot{\mathbf{w}}+\frac{\eta}{\kappa} \Upsilon * \dot{\mathbf{w}},
\end{array}\right.
$$

where $\mathbf{u}, \mathbf{U}$ and $\mathbf{w}=\phi(\mathbf{U}-\mathbf{u})$ are the solid displacement, the fluid displacement and the relative displacement vectors, respectively. I is the identity tensor, $\sigma$ is the stress tensor, $\varepsilon=1 / 2\left(\nabla \mathbf{u}+\nabla^{t} \mathbf{u}\right)$ is the strain tensor, and $p$ is the pore pressure. $\rho=\phi \rho_{f}+(1-\phi) \rho_{s}$ is the total density. The overlying dot denotes the derivative in terms of time $t$. $*$ stands for a convolution product in time. The quantity $\frac{\eta}{\kappa} \Upsilon * \dot{\mathbf{w}}$ corresponds to the time domain drag force between the porous skeleton and the pore fluid [12, 13]. Expression for $\Upsilon * \dot{\mathbf{w}}$ depends on the frequency range involved. In the low-frequency range, the relative motion between the fluid and the porous skeleton is of Poiseuille type, and then the expression is given by

$$
\Upsilon(t)=\delta(t) \Leftrightarrow \Upsilon(t) * \dot{\mathbf{w}}(t)=\dot{\mathbf{w}}(t)
$$

In the high-frequency range, the relative motion between the fluid and the porous skeleton is dominated by the inertial effects. We adopt here the wellknown model proposed by [14] and written in the frequency domain. The expression in the time domain is given in $[13,15]$ with the following form for 
the convolution product

$$
\Upsilon(t) * \dot{\mathbf{w}}(t)=\frac{1}{\sqrt{\Omega}}(D+\Omega)^{1 / 2} \dot{\mathbf{w}}(t)
$$

49

where $\Omega=\frac{\omega_{\mathrm{JKD}}}{\chi}$ with $\omega_{\mathrm{JKD}}=\frac{\eta \phi}{a \kappa \rho_{f}}$ the transition radial frequency between the low- and high-frequency ranges. $\chi$ is the Pride number. The operator $(D+\Omega)^{1 / 2}$ is a shifted order $1 / 2$ fractional derivative. Note that the drag force depends on the entire history of $\dot{\mathbf{w}}(\mathrm{t})$.

Pressure and stress components are eliminated from Eqs. (1a)-(1b) and substituted in Eqs. (1c)-(1d), giving a (u, w) second-order wave formulation $[9,10]$. By introducing the Helmholtz potentials for the solid $(\varphi, \Psi)$ and relative $\left(\varphi^{\mathrm{r}}, \boldsymbol{\Psi}^{\mathrm{r}}\right)$ displacements, the wave formulation yields a system of partial differential equations associated to these potentials as follows

$$
\left\{\begin{array}{l}
-\mu\left(\Delta \psi_{\theta}-\frac{\psi_{\theta}}{r^{2}}\right)+\left((1-\phi) \rho_{\mathrm{s}}+\phi \rho_{\mathrm{f}}\right) \ddot{\psi}_{\theta}+\rho_{\mathrm{f}} \ddot{\psi}_{\theta}^{\mathrm{r}}=0, \\
\rho_{\mathrm{f}} \ddot{\psi}_{\theta}+\frac{a \rho_{\mathrm{f}}}{\phi} \ddot{\psi}_{\theta}^{\mathrm{r}}+\frac{\eta}{\kappa} \frac{1}{\sqrt{\Omega}}(D+\Omega)^{1 / 2} \dot{\psi}_{\theta}^{\mathrm{r}}=0, \\
\left(\lambda+2 \mu+m \beta^{2}\right) \Delta \varphi+m \beta \Delta \varphi^{\mathrm{r}}-\rho \ddot{\varphi}-\rho_{\mathrm{f}} \ddot{\varphi}^{\mathrm{r}}=0, \\
m \beta \Delta \varphi+m \Delta \varphi^{\mathrm{r}}-\rho_{\mathrm{f}} \ddot{\varphi}-\frac{a \rho_{\mathrm{f}}}{\phi} \ddot{\varphi}^{\mathrm{r}}-\frac{\eta}{\kappa} \frac{1}{\sqrt{\Omega}}(D+\Omega)^{1 / 2} \dot{\varphi}^{\mathrm{r}}=0 .
\end{array}\right.
$$

Note that when projecting in the axisymmetric geometry, only the $\theta$ coordinate is useful for the vector potentials: $\boldsymbol{\Psi}=\psi_{\theta}(r, z, t) \mathbf{e}_{\theta}$.

For an axisymmetric configuration, it is relevant to introduce the $n$th order Hankel (or Fourier-Bessel) transform over the $r$ variable, and the Fourier transform over the $t$ variable, of an integrable function $f$, defined as follows [16]

$$
\tilde{f}_{n}(\xi)=\int_{0}^{+\infty} r f(r) J_{n}(\xi r) \mathrm{d} r \text { and } f^{*}(\omega)=\frac{1}{2 \pi} \int_{-\infty}^{+\infty} f(t) \mathrm{e}^{+\mathrm{i} \omega t} \mathrm{~d} t,
$$




$$
\widetilde{\varphi}_{0 j}^{\mathrm{r} *}(\xi, \omega)=\frac{\rho_{\mathrm{f}} \omega^{2}-m \beta\left(k_{\mathrm{P}_{j}}^{2}+\xi^{2}\right)}{m\left(k_{\mathrm{P}_{j}}^{2}+\xi^{2}\right)-\frac{a \rho_{\mathrm{f}} \omega^{2}}{\phi}-\mathrm{i} \frac{\eta \omega}{\kappa} \Upsilon^{*}(\omega)} \widetilde{\varphi}_{0 j}^{*}(\xi, \omega)={\widetilde{F_{j}}}^{*}(\xi, \omega) \widetilde{\varphi}_{0 j}^{*}(\xi, \omega),
$$

72 where $\widetilde{\boldsymbol{\Phi}}_{0}^{*}=\left\{\begin{array}{c}\widetilde{\varphi}_{0}^{*}(\xi, z, \omega) \\ \widetilde{\varphi}_{0}^{\mathrm{r} *}(\xi, z, \omega)\end{array}\right\}$, stiffness, mass and damping matrices being 
respectively

$$
\mathbf{K}_{\mathbf{P}}=\left[\begin{array}{cc}
\lambda+2 \mu+m \beta^{2} & m \beta \\
m \beta & m
\end{array}\right], \mathbf{M}=\left[\begin{array}{cc}
\rho & \rho_{\mathrm{f}} \\
\rho_{\mathrm{f}} & \frac{a \rho_{\mathrm{f}}}{\phi}
\end{array}\right], \mathbf{C}=\left[\begin{array}{cc}
0 & 0 \\
0 & \frac{\eta}{\kappa} \Upsilon^{*}(\omega)
\end{array}\right]
$$

From Eqs. (5) and (7), we introduce global wavenumbers $k_{\mathrm{S}}$ and $k_{\mathrm{P} j}$ by the relations $k_{\mathrm{S}}^{2}=k_{z \mathrm{~S}}^{2}+\xi^{2}=\frac{\omega^{2}}{\mu}\left((1-\phi) \rho_{\mathrm{S}}+\left(\phi+G^{*}(\omega) \rho_{\mathrm{f}}\right)\right)$ and $k_{\mathrm{P} j}^{2}=k_{z \mathrm{P} j}^{2}+$ $\xi^{2} . k_{z \mathrm{~S}}, k_{z \mathrm{P} j}$ and $\xi$ are the associated vertical wavenumbers and the radial wavenumber, respectively. Applying the Fourier transform over the $z$ variable defined as follows

$$
\bar{f}\left(k_{z}\right)=\frac{1}{2 \pi} \int_{-\infty}^{+\infty} f(z) \mathrm{e}^{-\mathrm{i} k_{z} z} \mathrm{~d} z
$$

to system (7), yields the dispersion relation when the determinant of matrix is equal to zero. Then, the general solution relative to the solid phase Helmholtz potentials of system (5)-(7) can be written as

$$
{\widetilde{\psi_{\theta 1}}}^{*}(\xi, z, \omega)={\widetilde{\psi_{\theta 1}}}^{* \mathrm{I}}(\xi, \omega) \mathrm{e}^{-\mathrm{i} k_{z \mathrm{~S}} z}+{\widetilde{\psi_{\theta 1}}}^{* \mathrm{R}}(\xi, \omega) \mathrm{e}^{\mathrm{i} k_{z \mathrm{~S}} z}
$$

$$
\begin{aligned}
\widetilde{\varphi}_{0}^{*}(\xi, z, \omega) & =\widetilde{\varphi}_{01}^{* \mathrm{I}}(\xi, \omega) \mathrm{e}^{-\mathrm{i} k_{z \mathrm{P}_{1}} z}+\widetilde{\varphi}_{01}^{* \mathrm{R}}(\xi, \omega) \mathrm{e}^{\mathrm{i} k_{z \mathrm{P}_{1}} z} \\
& +\widetilde{\varphi}_{02}^{* \mathrm{I}}(\xi, \omega) \mathrm{e}^{-\mathrm{i} k_{z \mathrm{P}_{2}} z}+\widetilde{\varphi}_{02}^{* \mathrm{R}}(\xi, \omega) \mathrm{e}^{\mathrm{i} k_{z \mathrm{P}_{2}} z},
\end{aligned}
$$

where I and R state the 'incident' (or downward) and the 'reflected' (or upward) waves, respectively.

The choice of an upward $(z)$ axis, implies that the conditions $\Im m\left\{k_{z \mathrm{~S}}\right\} \geq 0$ as well as $\Im m\left\{k_{z \mathrm{P}_{j}}\right\} \geq 0(j=1,2)$ should be satisfied to have a bounded field far away from the ground surface $(z \longrightarrow-\infty)$.

Besides, for an axisymmetric geometry

$$
{\widetilde{u_{r 1}}}^{*}(\xi, z, \omega)=-\xi \widetilde{\varphi}_{0}^{*}(\xi, z, \omega)-\frac{\partial{\widetilde{\psi_{\theta}}}^{*}}{\partial z}(\xi, z, \omega),
$$




$$
\widetilde{u}_{z 0}^{*}(\xi, z, \omega)=\frac{\partial \widetilde{\varphi}_{0}^{*}}{\partial z}(\xi, z, \omega)+\xi{\widetilde{\psi_{\theta}}}_{1}^{*}(\xi, z, \omega)
$$

Obviously, analogous expressions are obtained for the radial and vertical relative displacement components of vector w by substituting Helmholtz potentials for the solid displacement by relative ones.

Then, the expressions of ${\widetilde{u_{r}}}^{*}$ and ${\widetilde{u_{z}}}^{*}$ as functions of the scalar 'incident' and 'reflected' Helmholtz potentials, are obtained from Eqs. (10)-(11) substituted in Eqs. (12)-(13). The same developments are performed for ${\widetilde{w_{r}}}^{*}$ and ${\widetilde{w_{z}}}^{*}$ with relative Helmholtz potentials. In the present axisymmetric configuration, the exact stiffness matrix approach is based on vectors of transformed displacement and stress components $[9,10]$, defined as

$$
\widetilde{\mathbf{u}}^{*}=\left(\widetilde{u}_{r 1}^{*}, \mathrm{i}{\widetilde{u_{z 0}}}_{0}^{*}, \mathrm{i}{\widetilde{w_{z 0}}}^{*}\right)^{t}, \quad \widetilde{\Sigma}^{*}=\left({\widetilde{\sigma_{r z}}}^{*}, \mathrm{i}{\widetilde{\sigma_{z z}}}^{*},-\mathrm{i} \widetilde{p}_{0}^{*}\right)^{t}
$$

94

By using matrix notations, after setting $\widetilde{\boldsymbol{\Phi}}^{* \mathrm{I} / \mathrm{R}}=\left(\widetilde{\varphi}_{01}^{* \mathrm{I} / \mathrm{R}}, \widetilde{\varphi}_{02}^{* \mathrm{I} / \mathrm{R}},{\widetilde{\psi_{\theta}}}_{\theta_{1}}^{* \mathrm{I} / \mathrm{R}}\right)^{t}$, one can deduce

$$
\left\{\begin{array}{c}
\widetilde{\mathbf{u}}^{*}\left(\xi, z_{n-1}, \omega\right) \\
\widetilde{\mathbf{u}}^{*}\left(\xi, z_{n}, \omega\right)
\end{array}\right\}=\left[\begin{array}{cc}
\operatorname{Mat}^{\mathrm{I}} & \mathrm{Mat}^{\mathrm{R}} \mathrm{Z} \\
\operatorname{Mat}^{\mathrm{I}} \mathrm{Z} & \operatorname{Mat}^{\mathrm{R}}
\end{array}\right]\left\{\begin{array}{c}
\widetilde{\boldsymbol{\Phi}}^{\prime * \mathrm{I}}(\xi, \omega) \\
\widetilde{\boldsymbol{\Phi}}^{\prime * \mathrm{R}}(\xi, \omega)
\end{array}\right\},
$$

where $\widetilde{\boldsymbol{\Phi}}^{\prime * \mathrm{I} / \mathrm{R}}$ are modified potentials to have a better conditioning of Eq. (14) $[9] . \mathrm{Mat}^{\mathrm{I} / \mathrm{R}}=\left[\operatorname{mat}_{p q}^{\mathrm{I} / \mathrm{R}}\right] ; p=1,2,3 ; q=1,2,3$ with

$\begin{cases}\operatorname{mat}_{21}^{\mathrm{I}}=-\operatorname{mat}_{21}^{\mathrm{R}}=+k_{z \mathrm{P}_{1}} ; & \operatorname{mat}_{22}^{\mathrm{I}}=-\operatorname{mat}_{22}^{\mathrm{R}}=+k_{z \mathrm{P}_{2}} ; \\ \operatorname{mat}_{23}^{\mathrm{I} / \mathrm{R}}=+\mathrm{i} \xi ; & \operatorname{mat}_{31}^{\mathrm{I}}=-\operatorname{mat}_{31}^{\mathrm{R}}=+k_{z \mathrm{P}_{1}} \widetilde{F}_{1}^{*}(\xi, \omega) ; \\ \operatorname{mat}_{32}^{\mathrm{I}}=-\operatorname{mat}_{32}^{\mathrm{R}}=+k_{z \mathrm{P}_{2}}{\widetilde{F_{2}}}^{*}(\xi, \omega) ; & \operatorname{mat}_{33}^{\mathrm{I} / \mathrm{R}}=+\mathrm{i} \xi G^{*}(\omega) .\end{cases}$ 
102

$$
\begin{aligned}
{\widetilde{\sigma_{z z 0}}}^{*}(\xi, z, \omega) & =\left(\lambda+m \beta^{2}\right) \xi{\widetilde{u_{r 1}}}^{*}(\xi, z, \omega)+\left(\lambda+2 \mu+m \beta^{2}\right) \frac{\partial{\widetilde{u_{z}}}_{0}^{*}}{\partial z}(\xi, z, \omega) \\
& +m \beta \xi{\widetilde{w_{r}}}^{*}(\xi, z, \omega)+m \beta \frac{\partial{\widetilde{w_{z}}}^{*}}{\partial z}(\xi, z, \omega)
\end{aligned}
$$

103 104

$\mathrm{Z}=\operatorname{Diag}\left[\mathrm{e}^{\mathrm{i} k_{z \mathrm{P}_{1}} \mathrm{~h}_{n}}, \mathrm{e}^{\mathrm{i} k_{z \mathrm{P}_{2}} \mathrm{~h}_{n}}, \mathrm{e}^{\mathrm{i} k_{z \mathrm{~S}} \mathrm{~h}_{n}}\right]$ where Diag represents the terms of a diagonal matrix. $\mathrm{h}_{n}=z_{n-1}-z_{n}>0$ is the height of a specific layer " $n$ " bordered by the upper and the lower depth coordinates, $z_{n-1}$ and $z_{n}$, respectively.

Using the Biot behaviour law, stress components can be expressed in terms of transformed displacements

$$
\widetilde{\sigma_{r z 1}}(\xi, z, \omega)=\mu\left(\frac{\partial \widetilde{u_{r 1}}}{\partial z}(\xi, z, \omega)-\xi{\widetilde{u_{z}}}^{*}(\xi, z, \omega)\right),
$$

Besides, regarding the pore pressure, the equivalent of Eq. (1b) in the doubly transformed domain is

$$
\begin{aligned}
\widetilde{p}_{0}^{*}(\xi, z, \omega)= & -m\left\{\beta\left[\xi{\widetilde{u_{r}}}^{*}(\xi, z, \omega)+\frac{\partial{\widetilde{u_{z}}}^{*}}{\partial z}(\xi, z, \omega)\right]\right. \\
& \left.+\xi{\widetilde{w_{r}}}^{*}(\xi, z, \omega)+\frac{\partial{\widetilde{w_{z 0}}}^{*}}{\partial z}(\xi, z, \omega)\right\} .
\end{aligned}
$$

Then, the relation between stresses and Helmholtz potentials is given by

$$
\left\{\begin{array}{c}
\widetilde{\Sigma}^{*}\left(\xi, z_{n-1}, \omega\right) \\
-\widetilde{\Sigma}^{*}\left(\xi, z_{n}, \omega\right)
\end{array}\right\}=\left[\begin{array}{cc}
\mathrm{S}^{\mathrm{I}} & \mathrm{S}^{\mathrm{R}} \mathrm{Z} \\
-\mathrm{S}^{\mathrm{I} Z} & -\mathrm{S}^{\mathrm{R}}
\end{array}\right]\left\{\begin{array}{c}
\widetilde{\boldsymbol{\Phi}}^{\prime * \mathrm{I}}(\xi, \omega) \\
\widetilde{\boldsymbol{\Phi}}^{\prime * \mathrm{R}}(\xi, \omega)
\end{array}\right\}
$$

where $\mathrm{S}^{\mathrm{I} / \mathrm{R}}=\left[\begin{array}{c}\mathrm{I} / \mathrm{R} \\ \mathrm{s}_{p q}\end{array}\right] \quad ; \quad p=1,2,3 ; q=1,2,3 \quad$ with 


$$
108 \begin{cases}\mathrm{~s}_{11}^{\mathrm{I}}=-\mathrm{S}_{11}^{\mathrm{R}}=+2 \mathrm{i} \mu \xi k_{z \mathrm{P}_{1}} ; & \mathrm{s}_{12}^{\mathrm{I}}=-\mathrm{s}_{12}^{\mathrm{R}}=+2 \mathrm{i} \mu \xi k_{z \mathrm{P}_{2}} ; \\ \mathrm{s}_{13}^{\mathrm{I} / \mathrm{R}}=+\mu\left(k_{z \mathrm{~S}}^{2}-\xi^{2}\right) ; & \mathrm{s}_{21}^{\mathrm{I}}=\mathrm{s}_{21}^{\mathrm{R}}=-\mathrm{i}\left[\left(k_{z \mathrm{P}_{1}}^{2}+\xi^{2}\right)(\lambda+\right. \\ & \left.\left.m \beta^{2}+m \beta \widetilde{F}_{1}^{*}(\xi, \omega)\right)+2 \mu k_{z \mathrm{P}_{1}}^{2}\right] ; \\ \mathrm{s}_{22}^{\mathrm{I}}=\mathrm{s}_{22}^{\mathrm{R}}=-\mathrm{i}\left[\left(k_{z \mathrm{P}_{2}}^{2}+\xi^{2}\right)(\lambda+\right. & \\ \left.\left.m \beta^{2}+m \beta \widetilde{F}_{2}^{*}(\xi, \omega)\right)+2 \mu k_{z \mathrm{P}_{2}}^{2}\right] ; & \mathrm{s}_{23}^{\mathrm{I}}=-\mathrm{s}_{23}^{\mathrm{R}}=+2 \mu \xi k_{z \mathrm{~S}} ; \\ \mathrm{s}_{31}^{\mathrm{I} / \mathrm{R}}=-\mathrm{i} m\left(k_{z \mathrm{P}_{1}}^{2}+\xi^{2}\right)\left(\widetilde{F}_{1}^{*}(\xi, \omega)+\beta\right) ; & \\ \mathrm{s}_{32}^{\mathrm{I} / \mathrm{R}}=-\mathrm{i} m\left(k_{z \mathrm{P}_{2}}^{2}+\xi^{2}\right)\left(\widetilde{F}_{2}^{*}(\xi, \omega)+\beta\right) ; & \mathrm{s}_{33}^{\mathrm{I} / \mathrm{R}}=0 .\end{cases}
$$

110 Finally, analytical expressions for transformed displacement vectors are 111 written in condensed form as

$$
\left[\mathrm{T}_{\text {layer }_{n}}\right]_{6 \times 6}\left\{\begin{array}{c}
\widetilde{\mathbf{u}}^{*}\left(\xi, z_{n-1}, \omega\right) \\
\widetilde{\mathbf{u}}^{*}\left(\xi, z_{n}, \omega\right)
\end{array}\right\}=\left\{\begin{array}{c}
\widetilde{\boldsymbol{\Sigma}}^{*}\left(\xi, z_{n-1}, \omega\right) \\
-\widetilde{\mathbf{\Sigma}}^{*}\left(\xi, z_{n}, \omega\right)
\end{array}\right\} .
$$

112

A conventional assembling technique between the layers is then performed $[17,18]$. As $\left[\mathrm{T}_{\text {layer }_{n}}\right]$ is a $6 \times 6$ matrix, the global resulting matrix system has dimension $3(N+1) \times 3(N+1)$.

\subsection{Acoustic medium}

This part presents the analytical model formulation of wave propagation coming from an acoustic point source applied at $\mathrm{O}_{\mathrm{s}}$ (Fig. 1), in the water semi-infinite domain $\Omega_{0}$, characterized by celerity of waves $c$ and by density $\rho_{\mathrm{f}}$, assumed to be the same as in the porous media $\Omega_{n}$.

The acoustic equations are written as follows

$$
\left\{\begin{array}{l}
\ddot{\mathrm{U}}(r, z, t)=-\frac{1}{\rho_{\mathrm{f}}} \nabla p(r, z, t) \\
\Delta p(r, z, t)-\frac{1}{c^{2}} \ddot{p}(r, z, t)=-s(r, z, t)=-S(t) \delta\left(r-r_{\mathrm{s}}\right) \delta\left(z-z_{\mathrm{s}}\right)
\end{array}\right.
$$


where $p$ is the acoustic pressure, $\mathbf{U}$ is the fluid displacement and $s(r, z, t)$ is the impulse transient superpressure emitted from point $\mathrm{O}_{\mathrm{s}} . S(t)$ is a causal source term and $\delta$ is the Dirac function.

In the following, we introduce the fluid global wavenumber $k_{\mathrm{f}}$, linked to its vertical $\left(k_{z \mathrm{f}}\right)$ and radial $(\xi)$ components by $k_{\mathrm{f}}^{2}=k_{z \mathrm{f}}^{2}+\xi^{2}=\frac{\omega^{2}}{c^{2}}$, and use the mathematical property

$$
\widetilde{\delta}_{0}(\xi)=\int_{0}^{+\infty} r \delta(r) J_{0}(\xi r) \mathrm{d} r=\frac{1}{2 \pi} .
$$

Then, the partial differential equation relative to the pressure wave is obtained from the formulation of Eq. (20b) in the Fourier and 0th-order Hankel transform domain, as

$$
\frac{\partial^{2} \widetilde{p}_{0}^{*}}{\partial z^{2}}(\xi, z, \omega)+k_{z \mathrm{f}}^{2} \widetilde{p}_{0}^{*}(\xi, z, \omega)=-\frac{S^{*}(\omega)}{2 \pi} \delta\left(z-z_{\mathrm{s}}\right) .
$$

The solution to the above inhomogeneous equation results in the summation of two components:

- the complementary part $\widetilde{p_{\mathrm{C}}}{ }^{*}(\xi, z, \omega)$ corresponding to the solution of the homogeneous equation associated to Eq. (22),

- the principal part $\widetilde{p_{\mathrm{P} 0}}{ }^{*}(\xi, z, \omega)$ which is a specific solution of Eq. (22).

On the one hand, the complementary part of the solution is given by

$$
{\widetilde{p_{\mathrm{C}}}}_{0}^{*}(\xi, z, \omega)=\widetilde{\mathcal{P}}^{*}(\xi, \omega) \mathrm{e}^{\mathrm{i} k_{z \mathrm{f}} z}+\widetilde{\mathcal{Q}}^{*}(\xi, \omega) \mathrm{e}^{-\mathrm{i} k_{z \mathrm{f}} z},
$$

$\widetilde{\mathcal{P}}^{*}(\xi, \omega)$ and $\widetilde{\mathcal{Q}}^{*}(\xi, \omega)$ being the amplitudes, $\widetilde{\mathcal{Q}}^{*}(\xi, \omega)=0$ and $\Im m\left(k_{z \mathrm{f}}\right) \geqslant 0$ to satisfy the convergence condition when $z \longrightarrow+\infty$.

On the other hand, the calculation of the principal part of the solution is inspired by $[19,20]$. Concisely, the key steps are: 
(i) Setting the principal part of the solution as a simple Fourier integral expression

$$
{\widetilde{p_{\mathrm{P}} 0}}^{*}(\xi, z, \omega)=\int_{-\infty}^{+\infty} \mathcal{A}\left(\kappa_{z}\right) \mathrm{e}^{\mathrm{i} \kappa_{z} z} \mathrm{~d} \kappa_{z}
$$

(ii) Searching function $\mathcal{A}\left(\kappa_{z}\right)$ by introducting Eq. (24) in Eq. (22), multiplying the obtained equation by $\mathrm{e}^{-\mathrm{i} \kappa_{z} z}$ (where $\kappa_{z} \in \mathbb{R}$ ), integrating over the $z$ variable from $-\infty$ to $+\infty$, and using some mathematical properties of the Dirac function to obtain

$$
\mathcal{A}\left(\kappa_{z}\right)=-\frac{S^{*}(\omega)}{(2 \pi)^{2}} \frac{\mathrm{e}^{-\mathrm{i} \kappa_{z} z_{\mathrm{S}}}}{k_{z \mathrm{f}}^{2}-\kappa_{z}^{2}} .
$$

(iii) Rewriting the principal part of the solution gives

$$
{\widetilde{p_{\mathrm{P}}}}^{*}(\xi, z, \omega)=\frac{S^{*}(\omega)}{(2 \pi)^{2}} I\left(z-z_{\mathrm{s}}, k_{z \mathrm{f}}\right)
$$

where [19]

$$
I\left(z-z_{\mathrm{s}}, k_{z \mathrm{f}}\right)=\int_{-\infty}^{+\infty} \frac{\mathrm{e}^{\mathrm{i} \kappa_{z}\left(z-z_{\mathrm{s}}\right)}}{k_{z \mathrm{f}}^{2}-\kappa_{z}^{2}} \mathrm{~d} \kappa_{z}= \pm \mathrm{i} \pi \frac{\mathrm{e}^{\mathrm{i} \pm k_{z \mathrm{f}}\left|z-z_{\mathrm{s}}\right|}}{k_{z \mathrm{f}}} .
$$

(iv) Finally obtaining the single physically valid solution, satifying the Sommerfeld condition [20]

$$
{\widetilde{p_{\mathrm{P}}}}_{0}^{*}(\xi, z, \omega)=\frac{\mathrm{i} S^{*}(\omega)}{4 \pi} \frac{\mathrm{e}^{\mathrm{i} k_{z \mathrm{f}}\left|z-z_{\mathrm{s}}\right|}}{k_{z \mathrm{f}}} .
$$

Solution (28) corresponds to the one obtained in $[8,20]$. Indeed, these authors provide a Green's function as a solution of a cylindrical Helmholtz equation, which corresponds to the Fourier transform in time of Eq. (20b). Then, they calculate $\widetilde{p_{\mathrm{P}_{0}}^{*}}(\xi, z, \omega)$ by using a Sommerfeld integral decomposition of the simply transformed domain solution. 


\subsection{Interface equations}

The geometry under study leads to a set of interface equations along the $N$ plane interfaces $z_{n}(n=0, \cdots, N-1)$. For this purpose, we denote $[g]_{n}$ the jump in a function $g$ from $\Omega_{n}$ to $\Omega_{n+1}$ across $z_{n}$ as

$$
\begin{aligned}
{[g]_{n} } & =\lim _{\varepsilon \rightarrow 0, \varepsilon>0} g\left(r, z_{n}+\varepsilon, t\right)-\lim _{\varepsilon \rightarrow 0, \varepsilon>0} g\left(r, z_{n}-\varepsilon, t\right) \\
& =(g)_{n}^{+}-(g)_{n}^{-}
\end{aligned}
$$

- The porous / porous interfaces $z_{n}(n=1, \cdots, N-1)$ are assumed to be in perfect bonded contact [1]

$$
\begin{gathered}
{\left[u_{r}(r, z, t)\right]_{n}=0, \quad\left[u_{z}(r, z, t)\right]_{n}=0, \quad\left[w_{z}(r, z, t)\right]_{n}=0,} \\
{\left[\sigma_{r z}(r, z, t)\right]_{n}=0, \quad\left[\sigma_{z z}(r, z, t)\right]_{n}=0, \quad[p(r, z, t)]_{n}=0 .}
\end{gathered}
$$

- The fluid / porous interface $z_{0}=0$ is modeled with the following interface conditions $[1,6,7]$

$$
\left\{\begin{array}{l}
\left(u_{z}(r, z, t)\right)_{0}^{-}+\left(w_{z}(r, z, t)\right)_{0}^{-}=\left(U_{z}(r, z, t)\right)_{0}^{+}, \\
\left(\sigma_{r z}(r, z, t)\right)_{0}^{-}=0 \\
\left(\sigma_{z z}(r, z, t)\right)_{0}^{-}=-(p(r, z, t))_{0}^{+} \\
-[p(r, z, t)]_{0}=\frac{1}{\mathcal{K}}\left(\dot{w}_{z}(r, z, t)\right)_{0}^{-} .
\end{array}\right.
$$

where $\mathcal{K}$ is the hydraulic permeability of the interface. The case $\mathcal{K} \rightarrow+\infty$ describes open pores. For $\mathcal{K} \rightarrow 0$, Eq. (31d) is replaced by $\left(\dot{w}_{z}(r, z, t)\right)_{0}^{-}=$ 0 , stating sealed pores. An intermediate state for $\mathcal{K} \in] 0 ;+\infty[$ describes imperfect pores.

The formulation of the fluid / porous interface equations (31a)-(31c)-(31d) in the doubly transformed domain enables both to determine the amplitude 
$\widetilde{\mathcal{P}}^{*}(\xi, \omega)$ of the 'reflected' pressure wave in the fluid, and as a result to provide the following matrix block

$$
\begin{gathered}
{\left[\begin{array}{cc}
-\frac{\mathrm{i} \rho_{\mathrm{f}} \omega^{2}}{k_{z \mathrm{f}}} & -\frac{\mathrm{i} \rho_{\mathrm{f}} \omega^{2}}{k_{z \mathrm{f}}} \\
-\frac{\mathrm{i} \rho_{\mathrm{f}} \omega^{2}}{k_{z \mathrm{f}}} & -\frac{\mathrm{i} \rho_{\mathrm{f}} \omega^{2}}{k_{z \mathrm{f}}}-\frac{\mathrm{i} \omega}{\mathcal{K}}
\end{array}\right]\left\{\begin{array}{c}
\mathrm{i}\left(\widetilde{u}_{z 0}^{*}(\xi, z, \omega)\right)_{0}^{-} \\
\mathrm{i}\left(\widetilde{w}_{z 0}^{*}(\xi, z, \omega)\right)_{0}^{-}
\end{array}\right\}} \\
=\left\{\begin{array}{c}
-\mathrm{i}\left({\widetilde{\sigma_{z z}}}_{z}^{*}(\xi, z, \omega)\right)_{0}^{-}+\frac{S^{*}(\omega)}{2 \pi} \frac{\mathrm{e}^{\mathrm{i} k_{z \mathrm{f}} z_{\mathrm{s}}}}{k_{z \mathrm{f}}} \\
\mathrm{i}\left(\widetilde{p}_{0}^{*}(\xi, z, \omega)\right)_{0}^{-}+\frac{S^{*}(\omega)}{2 \pi} \frac{\mathrm{e}^{\mathrm{i} k_{z \mathrm{f}} z_{\mathrm{s}}}}{k_{z \mathrm{f}}}
\end{array}\right\} .
\end{gathered}
$$

Eq. (32) is assembled to Eq. (19) to give the radial and vertical solid and relative displacements at each interface. The transformed displacements, stresses, velocities and acoustic pressure everywhere inside each domain $\Omega_{n}$ can then be obtained analytically. The latter quantities are subsequently calculated in the spatio-temporal domain by means of inverse Hankel-Fourier transforms. For example, the acoustic or pore pressure can be written as follows

$$
p(r, z, t)=\int_{0}^{+\infty} 2 \Re e\left(\left\{\int_{0}^{+\infty} \xi \widetilde{p}_{0}^{*}(\xi, z, \omega) J_{0}(\xi r) \mathrm{d} \xi\right\} \mathrm{e}^{-\mathrm{i} \omega t}\right) \mathrm{d} \omega
$$

The integral over the radial wavenumber, which presents an oscillatory behavior due to factor $J_{0}(\xi r)$ and for which the envelope of the maximum amplitudes shows sharp peaks, is performed using an adaptive Filon quadrature, $[21,22]$. The adaptive procedure consists in dividing the entire interval into several parts based on what is known about $\widetilde{p}_{0}^{*}(\xi, z, \omega)$. Because of the sharp changes in the integrand occurring around the wavenumbers of the propagating waves, the wavenumbers are calculated and sorted out. The quadrature is performed by discretizing finely in their neighborhood and more coarsely 
farther away. The integral is truncated depending on the highest wavenumber and adapted to each frequency. As for the numerical processing over $\omega$, the quadrature is done using a Simpson scheme. Numerical values used for the various discretizations are those of [11].

\section{Results and discussion}

In this section, we propose firstly to validate the above theoretical formulation by using a half-space porous medium such as in [8]. To do that, we consider a limit test-case of the stratified configuration, composed of two layers presenting the same physical properties. Once the semi-analytical approach validated, we present new results coming from a seabed [23, 24] made of ten layers presenting various properties, as an extension of the half-space porous ground.

\subsection{Half-space test-case configuration}

Porous and fluid parameters are [8]: $\lambda=10.0 \times 10^{9} \mathrm{~Pa}, \mu=5.0 \times$ $10^{9} \mathrm{~Pa}, \rho_{\mathrm{s}}=2.5 \times 10^{3} \mathrm{~kg} \cdot \mathrm{m}^{-3}, \rho_{\mathrm{f}}=1.0 \times 10^{3} \mathrm{~kg} \cdot \mathrm{m}^{-3}, a=3, \beta=0.7$, $m=10.0 \times 10^{9} \mathrm{~Pa}, \phi=0.33, \eta=1.0 \times 10^{-3}$ Pa.s, $\kappa=10^{-8} \mathrm{~m}^{2}$ as well as,

$$
\Upsilon^{*}(\omega)=\left(1+\mathrm{i} \frac{\omega}{\Omega}\right)^{1 / 2}, \Omega=\frac{\eta \phi}{a \kappa \rho_{\mathrm{f}} \chi} \text { with } \chi=0.5[14]
$$

In the water domain $\Omega_{0}: \rho_{\mathrm{f}}=1.0 \times 10^{3} \mathrm{~kg} \cdot \mathrm{m}^{-3}, c=1414.0 \mathrm{~m} \cdot \mathrm{s}^{-1}$.

The emission point source is located at $z_{\mathrm{s}}=10 \mathrm{~m}$ and the observation ones are pointed by $r=20 \mathrm{~m}$ and $z= \pm 20 \mathrm{~m}$. Such as in [8], $S(t)$ is a Ricker wavelet and $S^{*}(\omega)$ its Fourier transform

$$
S(t)=\left(\left(1-2 \hat{\alpha}^{2}(t-\hat{\beta})^{2}\right) \mathrm{e}^{-\hat{\alpha}^{2}(t-\hat{\beta})^{2}} \text { and } \quad S^{*}(\omega)=\frac{\omega^{2}}{4 \hat{\alpha}^{3} \sqrt{\pi}} \mathrm{e}^{\left(\mathrm{i} \hat{\beta} \omega-\frac{\omega^{2}}{4 \hat{\alpha}^{2}}\right)},\right.
$$


where $\hat{\alpha}=\omega_{0} / 2$ and $\hat{\beta}=t_{\mathrm{s}}, \omega_{0}=2 \pi f_{0}\left(f_{0}=1.0 \times 10^{3} \mathrm{~Hz}\right)$ and $t_{\mathrm{s}}=2.5 \times$ $10^{-3} \mathrm{~s}$ being, respectively the central angular frequency and a shift in time.

As regards time evolution of pore pressure and fluid pressure, Fig. 2 shows that there is an excellent agreement between the results proposed by [8] and those obtained from our calculations. Both in permeable and impermeable cases, it checks the validity of the analytical approach in the half-space limit test-case.

\subsection{Extension to a stratified poroelastic seabed}

The regarded configuration is built from mechanical data taken from [23, 24]. It corresponds to a more realistic description of a seabed. To model the stratified ground and to illustrate the capabilities of our approach, we have chosen a ten layer geometry coupled to a half-space configuration. In the porous medium, the unchanged parameters are: $a=1.25, \rho_{\mathrm{s}}=2.65 \times$ $10^{3} \mathrm{~kg} \cdot \mathrm{m}^{-3}$ as well as the compressibility of the solid skeleton, $\chi_{\mathrm{s}}=36 \mathrm{GPa}$, and of the fluid volume, $\chi_{\mathrm{f}}=2 \mathrm{GPa}$. The Lamé constants are linked in this study by $\lambda=2 \mu$. Ranges of physical characteristics from the first layer to the half-space are: $\phi \in[0.5 ; 0.2], \kappa \in\left[10^{-9} ; 10^{-12}\right] \mathrm{m}^{2}$ and $\mu \in\left[10^{7} ; 10^{9}\right]$ Pa. From one stratum to another, only one of these three parameter values is modified as indicated in Tab. 1, as well as the related ones. The two Biot coefficients $\beta$ and $m$ are given by

$$
\beta=1-\frac{\chi_{\mathrm{o}}}{\chi_{\mathrm{s}}}, \frac{1}{m}=\frac{\beta-\phi}{\chi_{\mathrm{s}}}+\frac{\phi}{\chi_{\mathrm{f}}}, \quad \text { where } \chi_{\mathrm{o}}=\lambda+\frac{2}{3} \mu
$$

Parameters relative to the nature of the point source and to the water are the same as for the half-space test-case situation, the only differences are: $z_{\mathrm{s}}=5 \mathrm{~m}$ and the observation points are located at $r=1 \mathrm{~m}$ and $z= \pm 1 \mathrm{~cm}$ 


\begin{tabular}{|c|c|c|c|c|}
\hline Layer $n$ & $\begin{array}{c}\text { Height } \\
\mathrm{h}_{n}(\mathrm{~m})\end{array}$ & $\begin{array}{c}\text { Porosity } \\
\phi\end{array}$ & $\begin{array}{c}\text { Absolute } \\
\text { permeability } \kappa\left(\mathrm{m}^{2}\right)\end{array}$ & $\begin{array}{c}\text { Shear } \\
\text { modulus } \mu(\mathrm{Pa})\end{array}$ \\
\hline$n=1$ & 0.1 & 0.5 & $1 \times 10^{-9}$ & $1 \times 10^{7}$ \\
\hline$n=2$ & 0.1 & 0.5 & $1 \times 10^{-9}$ & $5 \times 10^{7}$ \\
\hline$n=3$ & 0.4 & 0.4 & $1 \times 10^{-9}$ & $5 \times 10^{7}$ \\
\hline$n=4$ & 0.4 & 0.4 & $5 \times 10^{-10}$ & $5 \times 10^{7}$ \\
\hline$n=5$ & 1.0 & 0.4 & $5 \times 10^{-10}$ & $1 \times 10^{8}$ \\
\hline$n=6$ & 1.0 & 0.3 & $5 \times 10^{-10}$ & $1 \times 10^{8}$ \\
\hline$n=7$ & 2.0 & 0.3 & $1 \times 10^{-11}$ & $1 \times 10^{8}$ \\
\hline$n=8$ & 5.0 & 0.3 & $1 \times 10^{-11}$ & $5 \times 10^{8}$ \\
\hline$n=9$ & 5.0 & 0.2 & $1 \times 10^{-11}$ & $5 \times 10^{8}$ \\
\hline$n=10$ & 10.0 & 0.2 & $1 \times 10^{-11}$ & $1 \times 10^{9}$ \\
\hline Half-space & $+\infty$ & 0.2 & $1 \times 10^{-12}$ & $1 \times 10^{9}$ \\
\hline
\end{tabular}

Table 1: Height of each layer and parameter values changing from one layer to another in the seabed

Firstly, Fig. 3 shows very similar time evolutions of fluid pressure at $z=1 \mathrm{~cm}$ observation height, both for sealed, imperfect and open pore interfaces between seawater and seabed. This means that the nature of the contact does not have any influence on the fluid pressure for the configuration under study. Secondly, considering only the impermeable cases, Fig. 3 emphasizes the fact that the properties of the first layer force the fluid 
pressure behaviour in the seabed when observation point is very close of the $z_{0}=0$ interface. Thirdly, central arrival time of the acoustic compressional wave is given by

$$
t_{\mathrm{f}}=\frac{\sqrt{\left(z_{\mathrm{s}}-z\right)^{2}+r^{2}}}{c}+t_{\mathrm{s}}=6.1 \mathrm{~ms} .
$$

214

In contrast, Fig. 4 clearly proves that the hydraulic permeability coefficient value has a strong impact on temporal variation of the vertical displacement at $z=-1 \mathrm{~cm}$ observation height, in the first layer of the seabed. Note that this trend is very attenuated when considering stresses in the seabed, not shown here.

Besides the comparison beween Fig. 4 and Fig. 5 highlights the multiple wave reflections at the porous / porous intefaces in the seabed. In addition, the confrontation between half-space and multilayered results yields higher differences in the intermediate case than those obtained in the extreme situations.

In the fourth layer, at $z=-80 \mathrm{~cm}$ observation height (Fig. 6), the nature of the contact does not have influence on the first displacement peaks any more. This time, the differences due to the kind of hydraulic interface is seen on the part of the response relative to the reflection waves $(t>9 \mathrm{~ms})$.

\section{Conclusions}

An axisymmetric model of wave propagation in poroelastic / acoustic configurations, has been presented, validated and extended by using a semianalytical method. The theoretical development has been based on a matrix 
block assembling technique for the porous layers and the fluid domain and takes into account various interface conditions. A half-space porous ground as a limit test-case of our multilayered medium has been considered to validate the analytical model. Indeed, regarding pore and fluid pressures, there is a very good agreement between the results coming from [8] and our calculations, whatever the pore nature. Then, the approach has been applied to a stratified seabed, as an extension of the half-space porous soil, providing new results which emphasize the variations in time of mechanical quantities.

From the obtained displacements and stresses, a future investigation consists in estimating mechanical and hydrological parameters of the systems under study. In parallel, the results could be compared to those issuing from finite difference, element and/or volume approaches or other analytical formulations such as transfer or transmission and reflexion matrices.

[1] T. Bourbié, O. Coussy, B. Zinszner, Acoustics of Porous Media, Gulf Publishing Company (1987).

[2] F.B. Jensen, W.A. Kuperman, M.B. Porter, H. Schmidt, Computational Ocean Acoustics, Springer (2011).

[3] J.F. Semblat, A. Pecker, Waves and Vibrations in Soils: Earthquakes, Traffic, Shocks, Construction Works, IUSS Press (2009).

[4] M. A. Biot, Theory of propagation of elastic waves in a fluid-saturated porous solid. I: Low-frequency range, Journal of the Acoustical Society of America, 28-2 (1956), 168-178.

[5] J. M. Carcione, Wave Fields in Real Media: Wave Propagation in 
Anisotropic, Anelastic, Porous and Electromagnetic Media, Elsevier (2007).

[6] B. Gurevich, M. Schoenberg, Interface conditions for Biot's equations of poroelasticity, Journal of the Acoustical Society of America, 105-5 (1999), 2585-2589.

[7] S. Feng, D. L. Johnson, High-frequency acoustic properties of a fluid / porous solid interface. I. New surface mode, Journal of the Acoustical Society of America, 74-3 (1983), 906-914.

[8] J. F. Lu, D. S. Jeng, Green's function for a harmonic acoustic point source within seawater overlying a saturated poroelastic seabed, Journal of Sound and Vibration, 307 (2007), 172-186.

[9] A. Mesgouez, G. Lefeuve-Mesgouez, Transient solution for multilayered poroviscoelastic media obtained by an exact stiffness matrix formulation, International Journal for Numerical and Analytical Methods in Geomechanics, 33 (2009), 1911-1931.

[10] G. Degrande, G. De Roeck, P. Van Den Broeck, D. M. J. Smeulders, Wave propagation in layered dry, saturated and unsaturated poroelastic media, International Journal of Solids and Structures, 35 (1998), 4753-4778.

[11] G. Lefeuve-Mesgouez, A. Mesgouez, G. Chiavassa, B. Lombard, Semianalytical and numerical methods for computing transient waves in 2D acoustic / poroelastic stratified media, Wave Motion, 49-7 (2012), $667-680$. 
[12] J.F. Lu, A. Hanyga, Fundamental solution for a layered porous half space subject to a vertical point force or a point fluid source, Computational Mechanics, 35 (2005), 376-391.

[13] E. Blanc, G. Chiavassa, B. Lombard, Biot-JKD model: Simulation of 1D transient poroelastic waves with fractional derivatives, Journal of Computational Physics, 237 (2013), 1-20.

[14] D. L. Johnson, J. Koplik, R. Dashen, Theory of dynamic permeability and tortuosity in fluid-saturated porous media. Journal of Fluid Mechanics, 176 (1987), 379-402.

[15] J.F. Lu, A. Hanyga, Wave field simulation for heterogeneous porous media with singular memory drag force, Journal of Computational Physics, 208 (2005), 651-674.

[16] M. Abramowitz, I. A. Stegun, Handbook of Mathematical Functions with Formulas, Graphs, and Mathematical Tables, 10th ed., National Bureau of Standards Applied Mathematics Series (1972).

[17] R.K.N.D. Rajapakse, T. Senjuntichai, Dynamic response of a multilayered poroelastic medium, Earthquake Engineering and Structural Dynamics, 24 (1995), 703-722.

[18] D.V. Jones, D. Le Houédec, M. Petyt, Ground vibrations due to a rectangular harmonic load, Journal of Sound and Vibration, 212-1 (1998), $61-74$.

[19] P. M. Morse et H. Feshbach, Methods of theoretical physics, Mc Graw Hill, New York (1953). 
[20] K. Aki, P. G. Richards, Quantitative Seismology : Theory and Methods I, San Francisco (1980).

[21] R.Barakat, E. Parshall, Numerical evaluation of the zero-order Hankel transform using Filon quadrature philosophy, Applied Mathematics Letters, 9-5 (1996), 21-26.

[22] R.Barakat, B.H. Sandler, Evaluation of the first-order Hankel transforms using Filon quadrature philosophy, Applied Mathematics Letters, 11-1 (1998), 127-131.

[23] R. D. Stoll, Reflection of acoustic waves at a water-sediment interface, Journal of the Acoustical Society of America, 70-1 (1981), 149-156.

[24] N. P. Chotiros, An inversion for Biot parameters in water saturated sand, Journal of the Acoustical Society of America, 112-5 (2002), 18531868. 


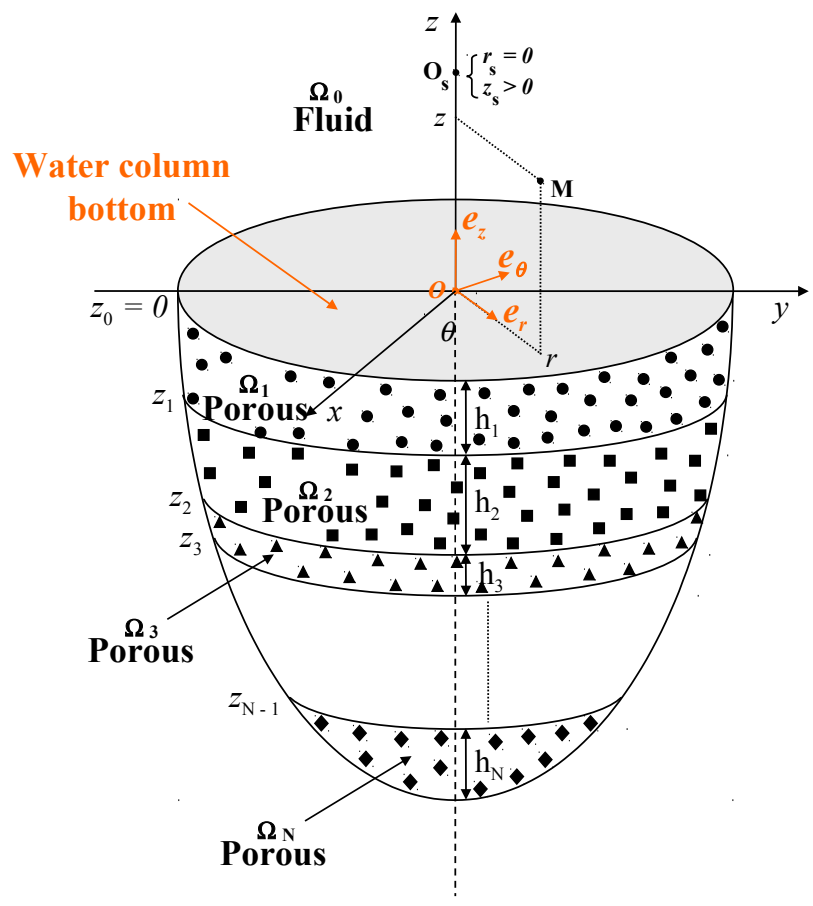

Figure 1: Axisymmetric geometry used for the multilayered poroelastic / acoustic media 


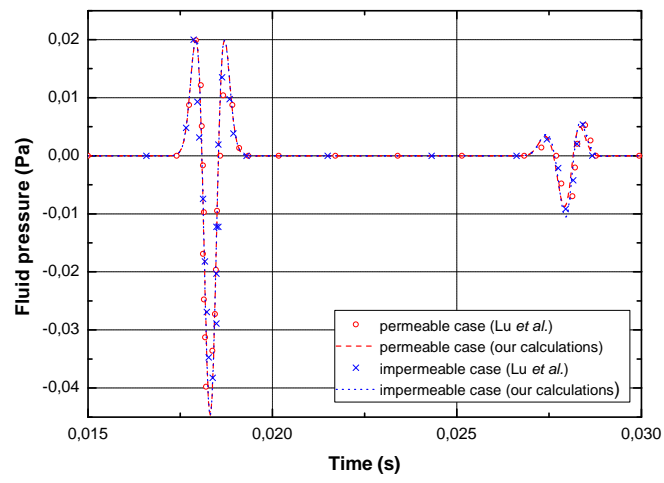

(a)

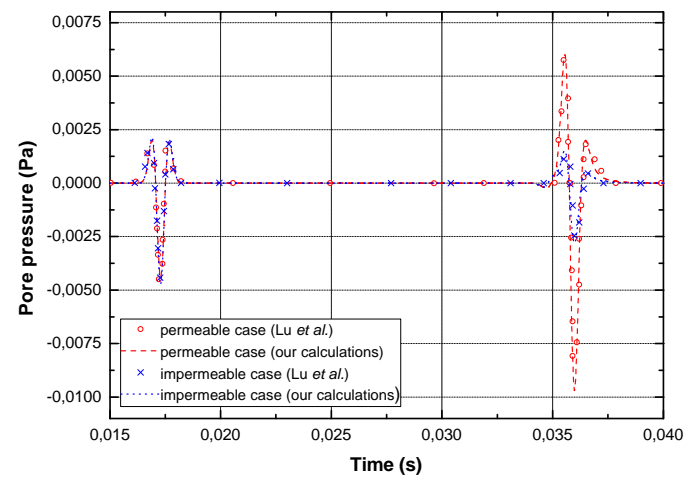

(b)

Figure 2: Time evolution of fluid pressure (up) and pore pressure (down) for permeable and impermeable cases, obtained by [8] and our calculations 


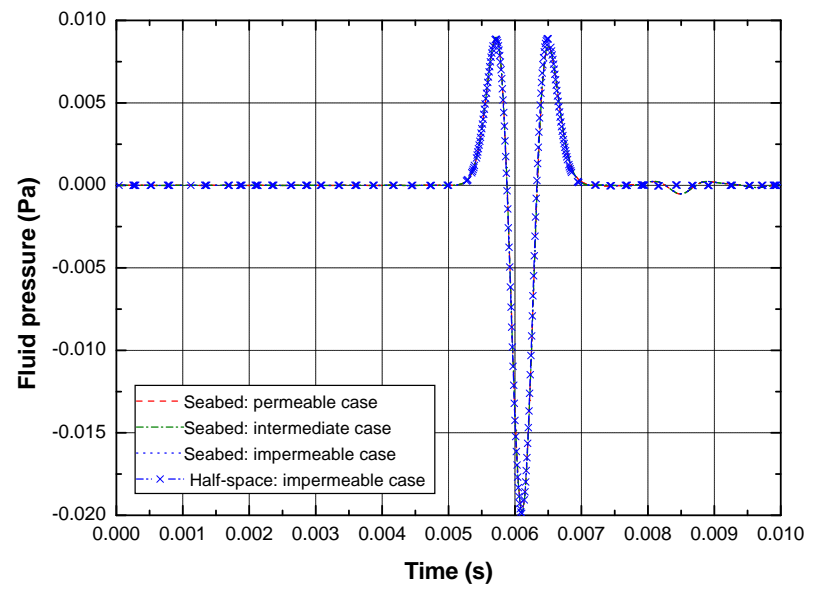

Figure 3: Time evolution of fluid pressure at $z=1 \mathrm{~cm}$ observation height, for permeable and intermediate $\left(\mathcal{K}=5 \times 10^{-10} \mathrm{~m} \cdot \mathrm{s}^{-1} \cdot \mathrm{Pa}^{-1}\right)$ cases in the seabed configuration, and for impermeable case in both the seabed and the half-space corresponding situation 


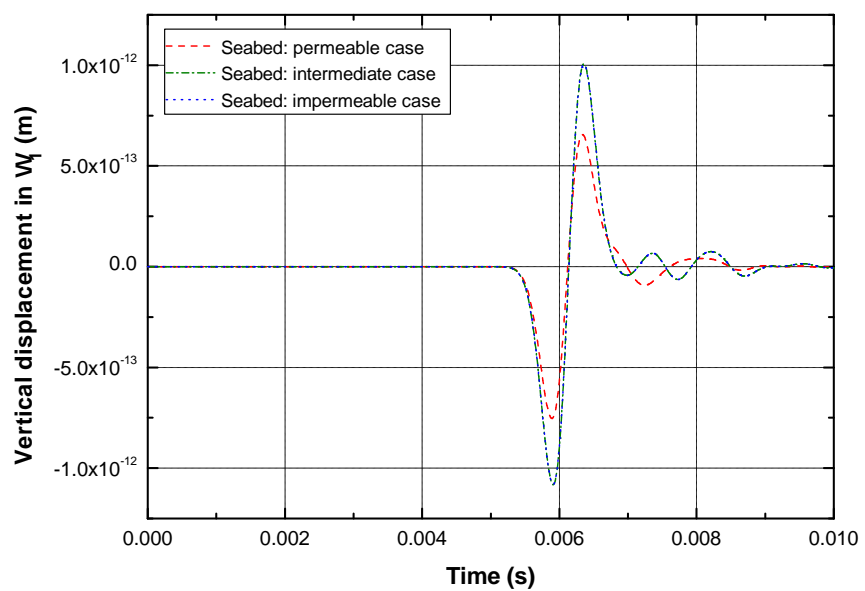

Figure 4: Time evolution of vertical displacement in the first layer at $z=-1 \mathrm{~cm}$ observation height, for permeable, intermediate $\left(\mathcal{K}=5 \times 10^{-10} \mathrm{~m} \cdot \mathrm{s}^{-1} \cdot \mathrm{Pa}^{-1}\right)$ and impermeable cases in the seabed configuration 


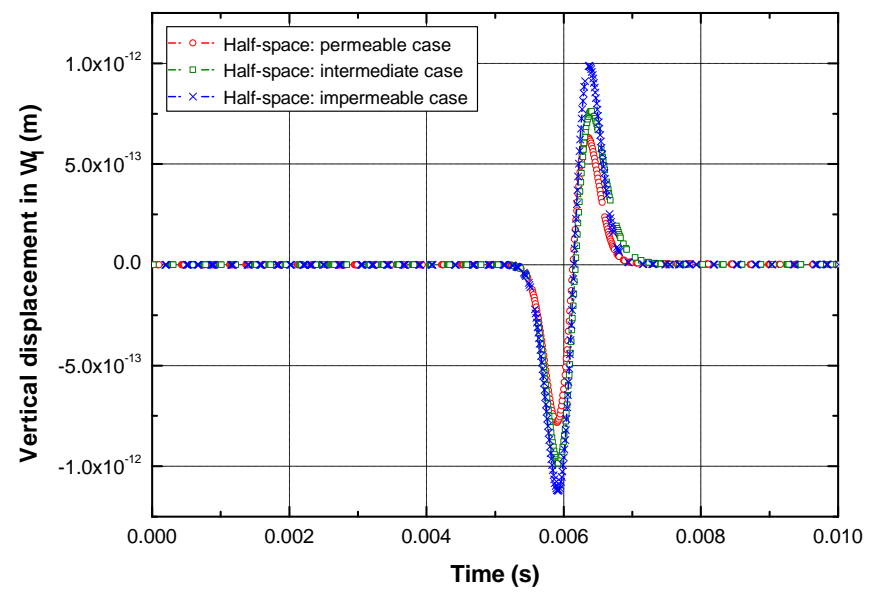

Figure 5: Time evolution of vertical displacement in the half-space corresponding situation to that of Fig. 3 


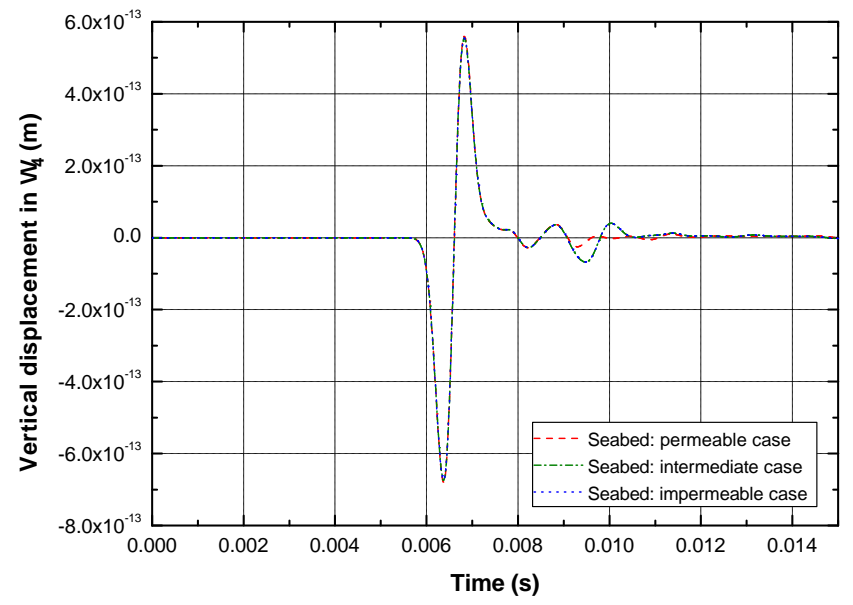

Figure 6: Time evolution of vertical displacement in the fourth layer at $z=-80 \mathrm{~cm}$ observation height, for permeable, intermediate $\left(\mathcal{K}=5 \times 10^{-10} \mathrm{~m} \cdot \mathrm{s}^{-1} \cdot \mathrm{Pa}^{-1}\right)$ and impermeable cases in the seabed configuration 